\title{
A NOTE ON THE QUOTIENT FIELD OF THE DOMAIN $D[[X]]$
}

\section{ROBERT GILMER}

If $D$ is an integral domain with quotient field $K$, it is well known that $K((X))$ may properly contain the quotient field of $D[[X]]$. We establish here necessary and sufficient conditions in order that $K((X))$ be the quotient field of $D[[X]]$.

Theorem 1. Suppose $L$ is the quotient field of $D[[X]]$. The following conditions are equivalent.

(1) $L=K((X))$.

(2) $K[[X]]=(D[[X]])_{N}$, where $N$ is the set of nonzero elements of $D$.

(3) If $\left\{\left(a_{i}\right)\right\}_{i=1}^{\infty}$ is a sequence of nonzero principal ideals of $D$, $\bigcap_{i=1}^{\infty}\left(a_{i}\right) \neq(0)$.

Proof. (1) $\rightarrow(3)$ : We suppose $K((X))=L$ and we consider a sequence $\left\{a_{i}\right\}_{i=0}^{\infty}$ of nonzero elements of $D$. For any nonnegative integer $k$, we define $c_{\kappa}=\left(a_{0} a_{1} \cdots a_{k}\right)^{-1}$. Then $c=\sum_{k=0}^{\infty} c_{k} X^{k} \in K[[X]] \subseteq L$, so that there is a nonzero element $b=\sum_{\mathbf{k}=0}^{\infty} b_{k} X^{k}$ of $D[[X]]$ such that $b c \in D[[X]]$. If the power series $b$ has order $r$, then $b / X^{r}$ $\in D[[X]]$ and $\left(b / X^{r}\right) c \in D[[X]]$, so we may assume that $r=0$ - that is, $b_{0} \neq 0$. Then if $m$ is a nonnegative integer, the coefficient of $X^{m}$ in $b c$ is

$$
\begin{aligned}
d & =\sum_{i=0}^{m} b_{i} c_{m-i} \\
& =\left(b_{0}+b_{1} a_{m}+b_{2} a_{m-1} a_{m}+\cdots+b_{m} a_{1} \cdots a_{m}\right) / a_{0} a_{1} \cdots a_{m} .
\end{aligned}
$$

By hypothesis, $d$ is in $D$, so that

$$
b_{0}+\left(b_{1}+b_{2} a_{m-1}+\cdots+b_{m} a_{1} \cdots a_{m-1}\right) a_{m}=d a_{0} a_{1} \cdots a_{m} \in\left(a_{m}\right) \text {. }
$$

Thus, $b_{0} \in\left(a_{m}\right)$ also. And since $m$ is arbitrary, $b_{0}$ is a nonzero element of $\bigcap_{i=0}^{\infty}\left(a_{i}\right)$ and (3) holds.

$(3) \rightarrow(2):$ We assume the validity of $(3)$. It is clear that $(D[[X]])_{N}$ $\subseteq K[[X]]$. We therefore consider an element $\xi=\sum_{n=0}^{\infty} \xi_{n} X^{n}$ where each $\xi_{n} \in K$. For each $n$, there is a nonzero element $b_{n}$ of $D$ such that $b_{n} \xi_{n} \in D$. Because (3) holds, there is a nonzero element $b$ in $\bigcap_{i=0}^{\infty}\left(b_{i}\right)$. For any $i$ we have $b=d_{i} b_{i}$ for some element $d_{i}$ of $D$. Hence $b \xi$ $=\sum_{n=0}^{\infty} d_{n}\left(b_{n} \xi_{n}\right) X^{n} \in D[[X]]$, so that $\xi=b \xi / b \in(D[[X]])_{N}$ and (2) holds.

Presented to the Society, November 11, 1966; received by the editors October 11, 1966. 
It is clear that $(2) \rightarrow(1)$.

REMARK 1. It may be readily seen that each of the following four conditions is equivalent to the conditions of the preceding theorem:

(4) $K[[X]] \subseteq L$;

(5) $K[[X]]$ is a quotient ring of $D[[X]]$;

(6) If $\left\{\left(a_{i}\right)\right\}_{i=1}^{\infty}$ is a decreasing sequence of nonzero principal ideals of $D, \bigcap_{i=1}^{\infty}\left(a_{i}\right) \neq(0)$;

(7) If $\left\{A_{i}\right\}_{1}^{\infty}$ is a sequence of nonzero ideals of $D$, then $\bigcap_{i=1}^{\infty} A_{i} \neq(0)$.

REMARK 2. From Theorem 1 , it is clear that $K[[X]][[Y]]$ has quotient field properly contained in $K((X))((Y))$. Hence the quotient field of $D[[X, Y]]$ is properly contained in $K((X))((Y))$, and this result generalizes to any number of indeterminates. But by using the methods of proof employed in Theorem 1, we can show that for any collection $\left\{X_{\alpha}\right\}$ of indeterminates over $D, D\left[\left[\left\{X_{\alpha}\right\}\right]\right]$ and $K\left[\left[\left\{X_{\alpha}\right\}\right]\right]$ have the same quotient field if and only if $D[[X]]$ and $K[[X]]$ have the same quotient field.

Example. In light of Theorem 1 it seems more reasonable to ask whether there exists a domain $D$, properly contained in its quotient field $K$, such that $K((X))$ is the quotient field of $D[[X]]$. The following example shows that the answer to this question is affirmative. In fact, we construct a valuation ring with this property. We let $A$ be a well-ordered set whose ordinal type is that of the set of ordinals preceding $\Omega$, the first uncountable ordinal, and we consider a collection $\left\{G_{\alpha}\right\}_{\alpha \in A}$ of groups, each isomorphic to the additive group of integers. We define $G$ to be the external weak direct sum of the groups $G_{\alpha}$. We think of the elements of $G$ as ordered $|A|$-tuples $(|A|$ is the cardinality of $A$ ) with integral coordinates, having only finitely many nonzero coordinates. $G$ becomes a totally-ordered abelian group if we define on $G$ the reverse lexicographic order: An element $g$ of $G$ is positive if the last nonzero coordinate of $g$ is a positive integer. The group $G$ is the value group of a valuation $v[1, \mathrm{p}$. 239]. If $V$ is the valuation ring of $v$ and if $K$ is the quotient field of $V, V \neq K$. We show $V[[X]]$ has quotient field $K((X))$ by showing that condition (6) of Remark 1 is fulfilled. Hence if $\left\{\left(a_{i}\right)\right\}_{i=1}^{\infty}$ is a decreasing sequence of principal ideals of $V$, then for any $i$ we have $v\left(a_{i}\right) \leqq v\left(a_{i+1}\right)$. Hence, by definition of the order relation on $G$, if the last nonzero entry of $v\left(a_{i}\right)$ is in $G_{\alpha_{i}}$, then $\alpha_{i}$ precedes or is equal to $\alpha_{i+1}$ for each positive integer $i$. By choice of the set $A$, there is an element $\alpha$ of $A$ such that each $\alpha_{i}$ precedes $\alpha$. It follows that there is an element $b$ of $V$ such that $v(b)>v\left(a_{i}\right)$ for each $i$ so that $b$ is a nonzero element of $\bigcap_{i=1}^{\infty}\left(a_{i}\right)$, and condition (6) holds. 
REMARK 3. Finally, we note that Theorem 1 and Remark 1 remain valid if the following changes are made in its statement: $D$ is a commutative ring with identity, and $K$ is the total quotient ring of $D$; nonzero principal ideals is replaced throughout by principal ideals generated by a regular element; nonzero ideals is replaced throughout by regular ideals; “ $\neq(0)$ " is replaced throughout by contains a regular element; and in (2), $N$ is taken to be the set of regular elements of $D$. Also, if $D$ is a domain satisfying any of the equivalent conditions of Theorem 1 , then any domain between $D$ and its quotient field has the same property.

\section{REFERENCES}

1. N. Jacobson, Lectures in abstract algebra. III, Van Nostrand, Princeton, N. J., 1964.

2. P. Jaffard, Theorie de la dimension dans les anneaux de polynomes, GauthierVillars, Paris, 1960.

Florida State University 